\title{
TEXTURE BASED ANALYSIS AND CONTOUR DETECTION OF CERVICAL SMEAR IMAGE
}

\author{
RamaPraba P.S ${ }^{1}$, Ranganathan H. $^{2}$ \\ ${ }^{1}$ Reserch scholar Sathyabama University, Tamil Nadu, India \\ ${ }^{2}$ Sakthimariamman Engg College, Tamil Nadu, India
}

\begin{abstract}
Pap smear is a very effective screening test for a cervical precancerous. However, hundreds of small slides have to be analyzed using microscope by the trained cytologist. This paper gives an overview of the state of the art and currently available literature and technique related Pap smear microscopic image analysis. Some techniques are used to detect cell components such as nuclei and cell boundaries. The following steps should be performed to extract the features and measure the area of the cells. Initially $\mathrm{ROI}$ of the denoised image undergoes segmentation to extract the features. In the next step the diameter of the nucleus was determined. The experimental results show that all the aforementioned techniques proposed have performed impressively based on the features extracted.
\end{abstract}

Keywords: Pap smear, segmentation, Region of interest (ROI), denoising, cytology.

\section{INTRODUCTION}

Cervical cancer is the second most commonly diagnosed cancer in women world wide exceeded only by the breast cancer [1]. Well known conventional method is Papanicolaou test, also called Pap smear or Pap test. It is a medical screening method that can help prevent cervical cancer. This is being replaced by faster and more efficient methods which use the latest and well developed technologies as the world is moving towards the glory of the information technology system.

The incidence and mortality related to cervical cancer can be reduced if this disease is detected at its precancerous state, known as squamous intraepithelial lesion (SIL)[2],[3].

Cervical cancer is a preventable disease, and unlike most other cancers it can be easily detected by a routine screening test, currently cervical smear screening is the most popular method used to detect the presence of abnormal cells developing in the cervix. The main purpose of the Pap smear is to detect for cell abnormalities that may occur from cervical cancer or before cancer develops. In Pap smear, sample cells are taken from the cervix using a small brush cotton wad stick or a wooden stick smeared on to thin glass plate ( slide). These slides are then delivered to laboratory where they are screened by cytologist.

The purpose of the smear screening is to diagnose prealignment cell changes before they progress to a cancer. Because the cervical cancer development is about 35 yrs so it is curable when we detect earlier .

Dysphasic cell are cells that have undergone precancerous changes they generally have longer and darker nuclei and have a tendency to doing together in large cluster .Mildly dysplastic cells have enlarged and bright nuclei. Moderately dyplastic cells have enlarged and bright nuclei. The nuclei may start to deteriorate which can be seen as a granulation of the nucleus server dyplastic cells have large, dark and often oddly shaped nuclei; their cytoplast is relatively dark and small [4].Hence precancers and cancers are associated with a variety of morphological and architectural alterations including size, texture and shape of cytoplast and nucleus, hyper chromasia and pleomorphsm and an increasing nuclear -cytoplastic ratio.

Current manual screening (Pap smear) methods are costly and are very likely to result inaccurate diagnoses due to human error (falsenegative and false positive). It should be borne in mind that the goal of automation based cervical cytology is that the percentage of normal samples that are classified as such must be as high as possible but getting an acceptable sensitivity degree.

To achieve this goal it has to be considered that the basic concepts underlying the pap test are morphological characteristics of nuclei and cytoplasm and nuclei/cytoplasm relation. 
In this paper we present part of our ongoing work towards automation of cervical smear screening process specifically based on the facts that in cytological studies nuclei are considered as the most informative regions and meaningful features.

\section{MICROSCOPIC IMAGES}

The pap smear slides usually contains both single cells and cluster of cells. Most of the cells are appear in high degree overlapping. The physical appearance of the cell in an images depends upon the specimen, which is collected from the cervix was smeared, stained and captured .The size of the image is depending upon the magnification lens used. The quality of the image is depending upon the resolution of the digital camera.

\section{A. Characteristic of the cervical cells}

In Pap smear slide along with pap strain some other strains like red blood cells white blood cells or even bacteria can appear.

Cervical cell consists of two main components nucleus and cytoplasm normally nucleus size is small and its shape is almost round to its infinity is darker than cytoplasm. The cervix cells which are taken from cervix mostly from columnar epithelium (CE )and squamous epithelium(SE).

\section{B. Pap smear cell Classification}

The microscope image of Pap smear image analysis[6], The SE consists of 4 layers of cells which are basal layer, parabasal layer, intermediate layer and superficial layer. Whereas the CE consists of only one layer of cells containing columnar cells and reserve cells. The columnar epithelium is located in the upper part of the cervix and the squamous epithelium in the lower part. Between these two is the metaplastic epithelium, also called the transformation zone or the squamocolumnar junction. It takes a skilled cyto technician to differentiate between the different kinds of cells and it is a time consuming job as every glass slide can contain up to 300,000 cells. The metaplastic epithelium consists of reserve cells from the columnar epithelium. The cell classes[6],[13] are mainly classified into 7 classes namely

1. Normal Columnar epithelial. Normal Super_cial squamous epithelial
2. Normal Parabasal squamous epithelial cells and Normal Intermediate squamous epithelial

3. Normal Intermediate squamous epithelial ells and Normal Columnar epithelial

4. Normal Super_cial squamous epithelial cells and Abnormal Mild squamous nonkeratinizing dysplasia,

5. Abnormal Mild squamous nonkeratinizing dysplasia, cells and Abnormal Moderate squamous nonkeratinizing dysplasia.

6. Abnormal Moderate squamous nonkeratinizing dysplasia cells and Abnormal Severe squamous nonkeratinizing dysplasia

7. Abnormal Severe squamous nonkeratinizing dysplasia,

By using these classification of the cell the nucleus appearance can visualized significant changes when the cell is affected by a disease, the identification and quantification of these changes contribute in the discrimination of normal and abnormal cells in Pap smear images.

\section{OUR APPROACH}

The principle objective of image preprocessing techniques is to process an image to make it more suitable than the original image for further application. Hence the image of the Pap smear slide are viewed under the microscope from which it is connected to the PC the slide are converted into digital images. The image are taken with variety of magnification present in a microscope like low magnification ,high magnification and oil magnification 10X,20X,40X for our approach we used high magnification (10X objective) because cell characteristic (nucleus and cytoplasm) can be viewed clearly. The image which converted into digital is consists of $640 \times 480$ pixels of Bit map file and totally of 307200 .

\section{A. Features of the cell}

The Pap smear cell is having several important features listed below

(a) Nucleus area

(b) Cytoplasm longest diameter

(c) Cytoplasm area

(d) Cytoplasm elongation 

size)

(e) N/C ratio (Size of nucleus relative to cell

(f) Cytoplasm roundness

(g) Nucleus brightness

(h) Nucleus perimeter

(i) Cytoplasm brightness

(j) Cytoplasm perimeter

(k) Nucleus shortest diameter

(l) Nucleus position

(m) Nucleus longest diameter

(n) Maxima in nucleus

(0) Nucleus elongation

(p) Minima in nucleus

(q) Nucleus roundness

(r) Maxima in cytoplasm

(s) Cytoplasm shortest diameter

(t) Minima in cytoplasm

For our work we taken only four features

\section{B. Segmentation:}

Cytological images is a fundamental subject of quantitative analysis because the normal and abnormal characteristics of cancer cells are all contain nucleus, so to isolate cytoplasm and nucleus is an important task of segmentation. There are general segmentation algorithm [5] of microscopic images have Common approaches for color image segmentation are clustering algorithms such spectral analyzer is used for these cell separation of nucleus and cytoplasm

\section{Classification of Cells}

The classification process for cervical cell image requires that single cells in the slides can be automatically isolated and analyzed. Several classification tech have been proposed. [6][9].

In feature extraction three different groups of parameters are extracted from cells. DensityOriented parameters are derived from the graylevel histogram calculated either over the nucleus or over the cytoplasm area. Shape oriented parameters are calculated from the gray level image but only over the nucleus area.

In cell classification, cells can be classified into two groups normal or atypical. The nucleus detection depending upon the 1) Surface Cell 2) Intermediate Cell and 3) Superficial Cells

The normal group is very in homogenous with respect to cytoplasm area, a very important cell characteristic this group includes both large surface cell and intermediate cell as well as the small cylinder cells. Therefore, here intermediate cell and surface cell were used.

In the smear classification, the smear is classified as normal or suspected malignant on the basis of the single cell classification, namely counting the numbers of atypical cells among normal cells.

In the First step images are reviewed at (10X objective) magnification. 50 normal cases and 50 abnormal cases of image size $480 \times 640$ were taken for analyzing in this paper. To separate the nucleus from cytoplasm the segmentation used spectral based clustering [10] .

Second step of the features extraction such as nucleus area and cytoplasm area from 50 normal images and 50 abnormal images were taken. The noise of the image is removed from this cell. After denoising, Region of Interest (ROI) is found which defines the actual size of the frame logged by the selected cropped part, measured with respect to the topleft corner of an image frame is selected and RGB images are converted into gray

scale images. Features of the nucleus perimeter standard deviation, areobtained by the following steps.

Step 1: Read Image

Step 2: Threshold the Image

Step 3: Remove the Noise

Step 4: Find the Boundaries

Step 5: Determine which Objects are Round The size and shape of the nucleus is round shape or ellipse hence and also darker intensity so that the image boundary is selected as the related to round 
shape The features are tabulated to obtain the mean value to analyze the pap smear.

\section{TABLE 1: MEAN VALUES FOR THE FEATURE EXTRACTED.}

\begin{tabular}{|c|c|c|}
\hline \multicolumn{1}{|c|}{ Mean } & SD & Diameter \\
\hline Normal Case & 52.5 & 0.19 \\
\hline Abnormal Case & 33.6 & 0.60 \\
\hline
\end{tabular}

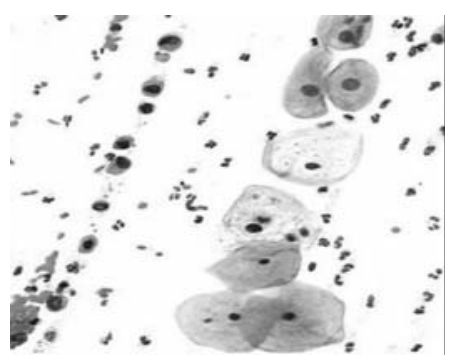

Fig. 1: Abnormal Pap smear image

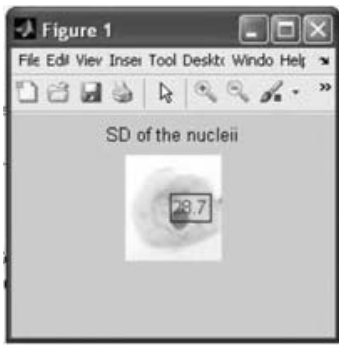

Fig. 2: SD of abnormal image.

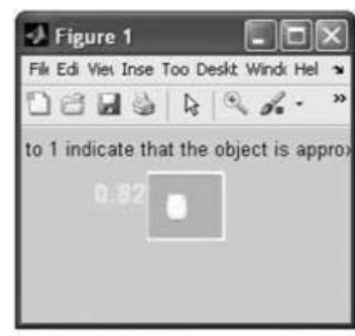

Fig. 3: Diameter of cropped nuclei.

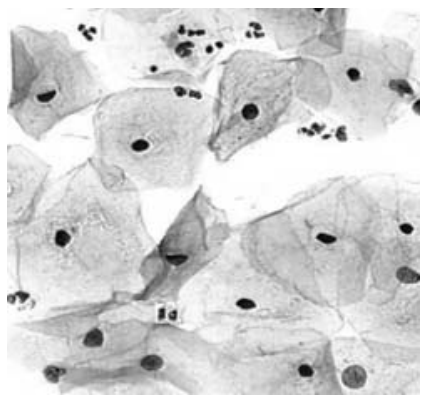

Fig. 4: Normal Pap Smear image.

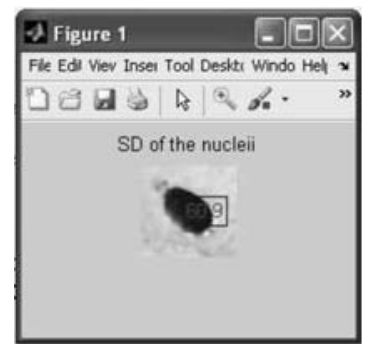

Fig. 5: SD of normal image.

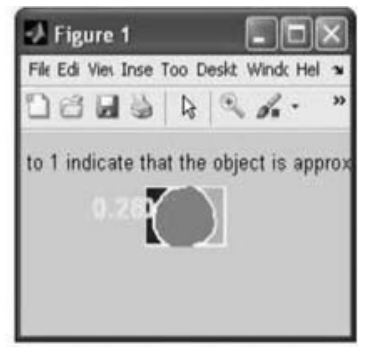

Fig. 6: Diameter of cropped nuclei.

\section{DISCUSSION AND FUTURE WORK}

The above figure shows the original image and feature extracted images. From the mean value obtained from the feature extraction, we analyzed that the SD value for normal cases is $>50$ and for abnormal cases it was $<40$. Similarly we analyzed that the diameter of nucleus of the normal case was around 0.20 microns and for abnormal cases $>0.50$ microns and it varies according to level of the cancer either low grade squamous intraepithelial lesion (LSIL)or High grade squamous intraepithelial lesion (HSIL). This proposed method classifies the cell based on the features and size of nuclei.

At present we are working towards to improve the methods and also extract the other feature from the digital images

\section{REFERENCES}

[1] D.M.Parkiu,F.Bray,J.Ferlayand P.Pisani " Global cancer statistics,2002” CAcancer J Clin,55(2), 2005, pp 74108.

[2] Kurman, R.J., Solomon D. The Bethesda System for reporting cervical/vaginal cytologic diagnoses, Springer Verlag, New York, 1994.

[3] Z. Ge, "Screening Pap smear with near infrared spectroscopy", Proc. Society for applied spectroscopy, vol. 49,no. 4, pp. 432436, 1995.

[4] E. Martin, "Papsmear classification," M.S. thesis, OerstedDTU, Automation, Tech. Univ. Denmark, Copenhagen, Denmark, 2003. 
[5] V. Dadeshidze, L. Olsson, and R. A. Domanik, "Segmentation of nuclear images in automated cervical cancer screening," Proc. SPIE-Int. Soc. Opt. Eng., vol. 2622, pp. 723- 727, 1995.

[6] Yannis Marinakis and George Dounias" Nearest Neighbor Based PapSmear Cell Classification Using Tabu Search for Feature Selection"Proc $f$ Nature inspired Smart Information system (NiSIS 2006) EU coordinate action ,Tenerife,spain,2006

[7] C. Duanggate, B. Uyyanonvara, and T. Koanantakul "A Review of Image Analysis and Pattern Classification Techniques for Automatic Pap Smear Screening Process" The 2008 International Conference on Embedded Systems and Intelligent Technology February 2729, 2008, Grand Mercure Fortune Hotel, Bangkok, Thailand

[8] Y. Marinakis and G. Dounias, "Nature inspired intelligent techniques for Pap smear diagnosis: Ant colony optimization for cell classification," Proc. Of
Nature inspired Smart Information Systems (NiSIS 2006), EU coordinate action, Tenerife, Spain, 2006.

[9] M. Kass, A. Witkin, and D. Terzopoulos, "Snakes: Active contour models," International Journal of Computer Vision, pp. 321331, 1987.

[10] "SelfTuning Spectral clustering " by Lihizalnikmanor and Pietraperena http://www.vision. caltech.edu/lihi/demos self tuning clustering html

[11] Jan Jantzen1, Jonas Norup, George Dounias, Beth Bjerregaard" Papsmear Benchmark Data For Pattern Classification" Technical University of Denmark: OerstedDTU, Automation,. 2003. [5] A. Meisels and C. Morin.

[12] Abnormal PAP Smears, HPV, Cervical Dysplasia and Cancer Edited excerpts from our book A Gynecologist's Second Opinion by WilliamH.Parker,M.D http://www.gynsecondopinion.com/pap.htm.

[13] "gynecologic cytopathology", Fadi W.Abdul karim,Nadia alKaisi 